\title{
Dashen's Phenomenon in Gauge Theories with Spontaneously Broken Chiral Symmetries
}

\author{
G. Akemann ${ }^{a}$, J.T. Lenaghan ${ }^{b}$ and K. Splittorff ${ }^{b}$ \\ a CEA/Saclay, Service de Physique Théorique, F-91191 Gif-sur-Yvette Cedex, France \\ ${ }^{b}$ The Niels Bohr Institute, Blegdamsvej 17, DK-2100 Copenhagen Ø, Denmark
}

We examine Dashen's phenomenon in the LeutwylerSmilga regime of QCD with any number of colors and quarks in either the fundamental or adjoint representations of the gauge group. In this limit, the theories only depend on simple combinations of quark masses, volume, chiral condensate and vacuum angle. Based upon this observation, we derive simple expressions for the chiral condensate and the topological density and show that they are in fact related. By examining the zeros of the various partition functions, we elucidate the mechanism leading to Dashen's phenomena in QCD.

\section{INTRODUCTION}

The vacuum angle, $\theta$, in QCD is experimentally constrained to be zero with a deviation of less than $10^{-9}$ 位. This amounts to a theoretical fine-tuning problem which is conceptually similar to the vanishing of the cosmological constant. Without an understanding of the physics at nonzero values of $\theta$, one will probably not be able to explain why $\theta$ is essentially zero. In this work, we approach the properties of the $\theta$-vacua from the vantage point of the hadronic phase where the physics is determined by the spontaneous breaking of chiral symmetry $(\mathrm{SB} \chi \mathrm{S})$. We consider QCD in the Leutwyler-Smilga regime [2] for which the volume, $L^{4}$, of Euclidean space-time is taken such that

$$
\frac{1}{\Lambda_{\mathrm{QCD}}} \ll L \ll \frac{1}{m_{\pi}} .
$$

The lower limit ensures that chiral perturbation theory is valid and that the Goldstone modes associated with $\mathrm{SB} \chi \mathrm{S}$ are the dominant degrees of freedom. The upper bound ensures that the Compton wavelength of the Goldstone modes is much greater than the size of the box and thus these modes can be treated as constant [3]. This has the advantage of allowing for exact, analytic calculations.

The various patterns of the $\mathrm{SB} \chi \mathrm{S}$ depend on both the number of colors, $N_{c}$, and the representation of the $N_{f}$ matter fields. They are simply codified by the Dyson index, $\beta$, which is assigned according to the antiunitary symmetry of the Euclidean Dirac operator [4. For $N_{c} \geq 3$ and matter in the fundamental representation $(\beta=2)$, the pattern of the $\mathrm{SB} \chi \mathrm{S}$ is given by $S U\left(N_{f}\right) \times S U\left(N_{f}\right) \rightarrow S U\left(N_{f}\right)$. Matter in the fundamental representation with $N_{c}=2(\beta=1)$ has the pattern $S U\left(2 N_{f}\right) \rightarrow S p\left(2 N_{f}\right)$. Finally, for any $N_{c}$ with matter in the adjoint representation $(\beta=4)$, the symmetry breaking pattern is given by $S U\left(N_{f}\right) \rightarrow S O\left(N_{f}\right)$.
At the level of the chiral QCD Lagrangian, the theory depends only on the combination $M e^{i \theta / N_{f}}$ where $M$ is the quark mass matrix [5]6]. In the Leutwyler-Smilga regime [2], this dependence is constrained even further. The quark masses, $m_{j}$, occur only in the rescaled combination $\mu_{j}=m_{j} V \Sigma$, where $V$ is the volume and $\Sigma$ is the chiral condensate in the chiral limit which is assumed to be non-vanishing. For one and two degenerate quark flavors Leutwyler and Smilga and Verbaarschot [2,7] found by explicit calculation that the exact partition function only depends on the combination $\mu \cos (\theta / 2)$.

One striking aspect of QCD at nonzero values of $\theta$ is the spontaneous breaking of $\mathrm{CP}$ in a first-order phase transition at $\theta=\pi$ known as Dashen's phenomenon [8]. In [5],6], this was shown by examining the $\theta$-dependence of the energy density of the large- $N_{c}$ chiral Lagrangian and was reconsidered more recently in [9 11] for different numbers of flavors and mass splittings. Going to the Leutwyler-Smilga regime, it was shown in Ref. 12] for $\beta=2$ that Dashen's phenomenon is indeed realized by directly calculating the discontinuity in the topological density at $\theta=\pi$. In this work, we extend 12 to $\beta=1$ and 4 by calculating the corresponding partition function, chiral condensate and topological density. We demonstrate that Dashen's phenomenon is generic to $\mathrm{QCD}$ regardless of the pattern of $\mathrm{SB} \chi \mathrm{S}$. By studying the zeros of the partition functions, we show that this is a direct consequence of the very restricted dependence of the partition function on quark masses, the chiral condensate and $\theta$. This restriction also establishes a direct relationship between the chiral condensate and the topological density as well as between their susceptibilities. The $N_{f}=2$ theories are sufficiently rich to illustrate the above points and as such we focus mainly on these cases. For a discussion of $N_{f}>2$, see the appendix.

\section{PARTITION FUNCTIONS}

In order to study Dashen's phenomenon for generic patterns of the $\mathrm{SB} \chi \mathrm{S}$, we first calculate the partition functions for $\beta=1$ and 4 including the contributions from all topological sectors. The full partition function as a function of $\theta$ may be decomposed into a sum over partition functions each of which is restricted to a fixed topological charge, $\nu$,

$$
\mathcal{Z}^{(\beta)}\left(\theta,\left\{\mu_{i}\right\}\right)=\sum_{\nu=-\infty}^{\infty} e^{i \nu \theta} \mathcal{Z}_{\nu}^{(\beta)}\left(\left\{\mu_{i}\right\}\right)
$$


Expressions at fixed $\nu$ were derived in Refs. 13 for $\beta=2$ and for degenerate masses with $\beta=1$ and 4 in Ref. [7]. Recently, the authors of Refs. [14,15 were able to calculate expressions for non-degenerate masses using chiral Random Matrix Theory ( $\chi \mathrm{RMT})$. The equivalence between $\chi$ RMT and QCD in the Leutwyler-Smilga regime has been established in a number of works (see Ref. 16. for a review of the literature). Using the results of 14, 15, we now calculate the full partition functions including the contributions from all topological sectors. Our normalization will be such that at vanishing masses $\mathcal{Z}^{(\beta)}(\theta,\{\mu=0\})=1$, which is only possible after summing over all topological charges [17]. The results for $N_{f}=1$ are known and are independent of $\beta$ [2, ,7]:

$$
\mathcal{Z}^{(\beta)}(\theta, \mu)=e^{\mu \cos \theta}
$$

For $\beta=4$, one must replace $\theta \rightarrow \theta / N_{c}$.

We begin with the first non-trivial case of two nondegenerate flavors. The same technique applies to $N_{f}>$ 2 where we refer to the appendix. The $\beta=1$ partition function with fixed topological charge is 14

$$
\begin{aligned}
\mathcal{Z}_{\nu}^{(1)}\left(\mu_{1}, \mu_{2}\right) & =8 \int_{0}^{1} d t t^{2}\left\{I_{\nu}\left(t \mu_{1}\right) \mu_{2} I_{\nu-1}\left(t \mu_{2}\right)\right. \\
& \left.-I_{\nu}\left(t \mu_{2}\right) \mu_{1} I_{\nu-1}\left(t \mu_{1}\right)\right\}
\end{aligned}
$$

In order to sum over all topological charges we use the identity

$$
\begin{aligned}
& \sum_{\nu=-\infty}^{\infty} e^{i \nu \theta} I_{\nu+n}\left(\mu_{1}\right) I_{\nu+m}\left(\mu_{2}\right)= \\
& =e^{-i n \theta}\left(\frac{\mu_{12}(\theta)}{\mu_{1} e^{-i \theta}+\mu_{2}}\right)^{n-m} I_{n-m}\left(\mu_{12}(\theta)\right),
\end{aligned}
$$

where a reduced mass is defined as

$$
\mu_{12}(\theta) \equiv \sqrt{\mu_{1}^{2}+\mu_{2}^{2}+2 \mu_{1} \mu_{2} \cos \theta}
$$

Performing the summation over $\nu$ and calculating the integral over $t$, the two flavor partition function for $\beta=1$ becomes

$$
\mathcal{Z}^{(1)}\left(\theta, \mu_{1}, \mu_{2}\right)=8 \frac{I_{2}\left(\mu_{12}(\theta)\right)}{\mu_{12}(\theta)^{2}} .
$$

Let us stress the striking similarity to $\beta=2$ where 17,12

$$
\mathcal{Z}^{(2)}\left(\theta, \mu_{1}, \mu_{2}\right)=2 \frac{I_{1}\left(\mu_{12}(\theta)\right)}{\mu_{12}(\theta)}
$$

At $\theta=\pi$, note that $\mu_{12}(\theta=\pi)=\left|\mu_{1}-\mu_{2}\right|$. To take the limit of degenerate quark masses, $\mu_{1}=\mu_{2}=\mu$, we simply replace $\mu_{12}(\theta)=2 \mu\left|\cos \frac{\theta}{2}\right|$ in eqn. (2.6). This agrees with the results from Ref. [7]. At $\theta=\pi$, we find $\mathcal{Z}^{(1)}(\theta=\pi, \mu, \mu)=1$, i.e. the partition function is independent of quark masses and volume just like the partition function for $\beta=2$ [10,12]. In Ref. [10], this was shown to be due to a cancelation of terms at lowest order in chiral perturbation theory and higher-order terms were considered. In the Leutwyler-Smilga scaling regime, however, these terms are suppressed.

As discussed in Ref. [2], the allowed topological charges in adjoint QCD, $\beta=4$, are rescaled by a factor of $N_{c}$, so $\nu=\bar{\nu} / N_{c}$ where $\bar{\nu}$ is an integer. The partition function thus only depends on the combination $M e^{i \theta /\left(\bar{N}_{f} N_{c}\right)}$. The number of Majorana fermions is given by $\bar{N}_{f}$ and we restrict the present discussion to $\bar{N}_{f}=2$. The partition function with fixed topological charge can be calculated following [7]:

$$
\begin{aligned}
& \mathcal{Z}_{\nu}^{(4)}\left(\mu_{1}, \mu_{2}\right)=\int_{0}^{2 \pi} \frac{d b}{4 \pi} \sum_{n=-\infty}^{+\infty} \frac{1}{2 n+1} \\
& \times\left\{I_{\bar{\nu}+n}(\mu(b)) I_{\bar{\nu}-n}(\eta(b))+I_{\bar{\nu}-n}(\mu(b)) I_{\bar{\nu}+n}(\eta(b))\right\}
\end{aligned}
$$

with

$$
\begin{aligned}
& \mu(b)=\mu_{1} \cos (b)^{2}+\mu_{2} \sin (b)^{2}, \\
& \eta(b)=\mu_{2} \cos (b)^{2}+\mu_{1} \sin (b)^{2} .
\end{aligned}
$$

Although the full partition function can be obtained from (2.8) (see eqn. (A3)), we only treat the degenerate mass case for simplicity in what follows. The partition function (2.8) then drastically simplifies to

$$
\mathcal{Z}_{\nu}^{(4)}(\mu, \mu)=\int_{0}^{1} d t I_{2 \bar{\nu}}(2 t \mu) .
$$

This can be seen by applying $\partial_{\mu} \mu$ to eqn. (2.8) at equal mass $\mu$ and then integrating back. In order to compute the summation over the topological charges $\bar{\nu}$, it is useful to split the generating function for $I_{\nu}(x)$ into odd and even parts:

$$
\sum_{\nu=-\infty}^{\infty} t^{2 \nu} I_{2 \nu}(x)=\cosh \left[\frac{x}{2}\left(t+t^{-1}\right)\right],
$$

and similarly for the odd contribution. With the help of this identity and after integrating over $t$, we obtain

$$
\mathcal{Z}^{(4)}(\theta, \mu, \mu)=\frac{\sinh \left(2 \mu \cos \frac{\theta}{2}\right)}{2 \mu \cos \frac{\theta}{2}},
$$

which was first derived in Ref. [2]. At $\theta=\pi$, the partition function is again equal to unity and thus is independent of quark masses and volume. This is again an indication that there are dominant terms at next-to-leading order in chiral perturbation theory. For $\bar{N}_{f}>2$ we refer to the appendix.

\section{TOPOLOGICAL DENSITY AND CHIRAL CONDENSATE}

The chiral condensate and the topological density are defined as 


$$
\begin{aligned}
\Sigma_{j}^{(\beta)}\left(\theta,\left\{\mu_{i}\right\}\right) & \equiv \Sigma \frac{\partial}{\partial \mu_{j}} \log \mathcal{Z}^{(\beta)}\left(\theta,\left\{\mu_{i}\right\}\right), \\
\sigma^{(\beta)}\left(\theta,\left\{\mu_{i}\right\}\right) & \equiv-\frac{1}{V} \frac{\partial}{\partial \theta} \log \mathcal{Z}^{(\beta)}\left(\theta,\left\{\mu_{i}\right\}\right) .
\end{aligned}
$$

In the case where $\mathcal{Z}$ is restricted to be a function of only some combination of $\theta$ and $\left\{m_{i}\right\}$, e.g. $X\left(\theta, \mu_{1}, \mu_{2}\right)=$ $\mu_{12}(\theta)$, we readily find that

$$
\frac{\Sigma_{j}^{(\beta)}\left(\theta,\left\{\mu_{i}\right\}\right)}{\sigma^{(\beta)}\left(\theta,\left\{\mu_{i}\right\}\right)}=-V \Sigma \frac{\partial_{\mu_{j}} X}{\partial_{\theta} X} .
$$

While this relation is a simple consequence of the observation that $\mathcal{Z}=\mathcal{Z}(X)$, it establishes a deep relationship between the chiral and the topological properties of the vacuum. The chiral and the topological susceptibility are also related although they are no longer proportional.

The $N_{f}=1$ case is special since the entire chiral symmetry group is explicitly broken by the axial anomaly and there is no spontaneous breaking of any symmetry. As a result, there are no Goldstone modes. The topological density is

$$
\sigma^{(\beta)}(\theta, \mu)=\frac{\mu}{V} \sin \theta,
$$

for $\beta=1,2$ and 4 (with $\theta \rightarrow \theta / N_{c}$ ) and there is no discontinuity at $\theta=\pi$. This is another indication of the interplay between the $\mathrm{SB} \chi \mathrm{S}$ and the topological properties of the theory.

Turning to the more interesting case of two degenerate flavors we only have to calculate partial derivatives of the variable $X=2 \mu\left|\cos \frac{\theta}{2}\right|$ and of the corresponding partition function. For $\beta=1$ we obtain

$$
\Sigma^{(1)}(\theta, \mu, \mu)=\Sigma \frac{X}{\mu} \frac{I_{3}(X)}{I_{2}(X)}
$$

and thus

$$
\sigma^{(1)}(\theta, \mu, \mu)=\frac{1}{V} \frac{\mu^{2} \sin \theta}{X} \frac{I_{3}(X)}{I_{2}(X)} .
$$

The similarity with $\beta=2$ in [12] is again striking. To recover their result we merely have to replace the logarithmic derivative $\partial_{X} \log \mathcal{Z}(X)$ leading to $I_{2}(X) / I_{1}(X)$ instead of $I_{3}(X) / I_{2}(X)$.

For $\beta=4$ we obtain from eqn. (2.12)

$$
\begin{aligned}
\Sigma^{(4)}(\theta, \mu, \mu) & =\Sigma \frac{X \cosh X-\sinh X}{\mu \sinh X}, \\
\sigma^{(4)}(\theta, \mu, \mu) & =\frac{1}{V} \frac{\mu \sin \frac{\theta}{2}}{X} \frac{X \cosh X-\sinh X}{\sinh X} .
\end{aligned}
$$

For all three values of $\beta$ the quantities $\Sigma^{(\beta)}(\theta, \mu, \mu)$ and $\sigma^{(\beta)}(\theta, \mu, \mu)$ vanish at $\theta=\pi$. Furthermore, $\sigma^{(\beta)}(\theta, \mu, \mu)$ exhibits a discontinuity $\sim \operatorname{sign}\left(\cos \frac{\theta}{2}\right)$ at $\theta=\pi$ for large scaling variable, $\mu \gg 1$. This follows from $\lim _{X \rightarrow \infty} I_{\nu+1}(X) / I_{\nu}(X)=\operatorname{sign}(X)$ for $\beta=1,2$ and similarly from eq. (3.8) for $\beta=4$ (for $\beta=2$ this was shown already in [12]). Thus Dashen's phenomenon is generic for all $\mathrm{SB} \chi \mathrm{B}$ patterns with $N_{f}=2$.

The result for non-degenerate flavors follows in a similar fashion with $X=\mu_{12}(\theta)$. For $\beta=1$ and 2, the chiral condensate obtained from eqs. (2.6) and (2.7) respectively is easily seen to vanish at $\theta=\pi$. The case $\beta=4$ is slightly more involved and we refer to the appendix (see eqs. (A4) and (A5)). To see the phase transition in $\sigma^{(\beta)}\left(\theta, \mu_{1}, \mu_{2}\right)$ for $\beta=1,2$ one must take the limit $\mu_{1}, \mu_{2} \rightarrow \infty$ such that

$$
\lim _{\mu_{1}, \mu_{2} \rightarrow \infty} \frac{\left(\mu_{1}-\mu_{2}\right)^{2}}{\mu_{1} \mu_{2}} \rightarrow 0 .
$$

This leads to $\mu_{12}(\theta) \rightarrow 2 \sqrt{\mu_{1} \mu_{2}}\left|\cos \frac{\theta}{2}\right|$ and thus Dashen's phenomenon occurs in the same way as for degenerate masses. For $\beta=2$ the bound (3.9) is consistent with the one found in 55. We obtain a discontinuity in $\sigma^{(\beta)}$ at $\theta=\pi$ and the vanishing of the chiral condensate for both degenerate and non-degenerate masses. Hence, these properties are not particular to the degenerate mass case. We expect that Dashen's phenomenon also happens for $N_{f}>2$ for all three values of $\beta$. It has been shown in [12] to occur for $N_{f}=3$ degenerate flavors and for $\beta=4$ with four flavours it follows from eq. (A7).

\section{ZEROS OF THE PARTITION FUNCTIONS}

We now consider the zeros of the various partition functions in the Leutwyler-Smilga regime 1 . Using the theorems of Yang and Lee [19] we expose the mechanism leading to Dashen's phenomenon in the Leutwyler-Smilga regime for all $\beta$. Yang and Lee proved that the analytic behavior of the partition function, $\mathcal{Z}$, is determined completely by its zeros when the real parameters of the theory are continued into the complex plane. The nonanalytic behavior of $\mathcal{Z}$ as function of the real-parameters occurs where the zeros in the complex plane pinch the real axis. The non-analyticity of the partition function is tantamount to the existence of a phase transition. The $\mathrm{SB} \chi \mathrm{S}$ in $\mathrm{QCD}$ occurs when the zeros in complex quark mass plane pinch the real $\mu$-axis at $\mu=0$. Dashen's phenomenon manifests itself as the zeros in the complex $\theta$-plane pinch the real $\theta$-axis. We note that since there is no spontaneous breaking of chiral symmetry for $N_{f}=1$, there is no solution to the equation $\mathcal{Z}^{\beta, N_{f}=1}(\theta, \mu)=0$ as is easily verified using (2.2).

For illustrative purposes, we next take two degenerate flavors and any value of $\beta$ and so $\mathcal{Z}^{(\beta)}(\theta, \mu, \mu)=$ $\mathcal{Z}^{(\beta)}\left(2 \mu \cos \frac{\theta}{2}\right)$. Consider the analytic continuation of $X \equiv 2 \mu \cos \frac{\theta}{2}$ into the complex plane. For all three $\beta$ 's

\footnotetext{
${ }^{1}$ In Ref. 18, an analysis of the zeros was performed for the $\chi \mathrm{RMT}$ partition function of $\beta=2$ at fixed topological charge.
} 
the equation $\mathcal{Z}^{(\beta)}\left(X_{j}\right)=0$ is solved by purely imaginary values of $X_{j}$. The solutions are the non-vanishing zeros of $I_{2}, I_{1}$, and $\sinh$ for $\beta=1,2,4$ respectively. Given the purely imaginary zeros, $X_{j}$, in the complex $X$-plane, it is simple to find the zeros, $\mu_{j}$, in the complex $\mu$-plane given a real $\theta$

$$
\mu_{j}=\frac{X_{j}}{2 \cos \frac{\theta}{2}}
$$

and the zeros, $\theta_{j} \equiv a_{j}+i b_{j}$, in the complex $\theta$-plane given a real $\mu$

$$
a_{j}=\pi \text { and } b_{j}=2 \operatorname{arcsinh}\left(\frac{i X_{j}}{2 \mu}\right) .
$$

From this we observe that $\mu_{j} \rightarrow \infty$ for $\theta \rightarrow \pi$, indicating the independence of the partition function on $\mu$ at $\theta=\pi$. Furthermore we see that $\theta_{j}=\pi+i b_{j} \rightarrow \pi$ for $\mu \rightarrow \infty$, indicating Dashen's phenomenon. We stress that this is independent of $\beta$. If we consider two non-degenerate flavors, then for $\beta=1$ and 2 we have $\mathcal{Z}^{(\beta)}\left(\theta, \mu_{1}, \mu_{2}\right)=$ $\mathcal{Z}^{(\beta)}\left(\mu_{12}(\theta)\right)$. Repeating the above argument we see that we have exactly the same zeros, but in this case $X \equiv$ $\mu_{12}(\theta)$. Solving this for $\theta_{j}$ at fixed real $\mu_{1}, \mu_{2}>0$ one finds that

$$
a_{j}=\pi \text { and } b_{j}=\operatorname{arccosh}\left(\frac{\mu_{1}^{2}+\mu_{2}^{2}-X_{j}^{2}}{2 \mu_{1} \mu_{2}}\right) .
$$

We observe that $\theta_{j}$ moves into the vicinity of $\pi$ as $\mu_{1}, \mu_{2} \rightarrow \infty$ provided that the bound (3.9) holds, in complete accordance with what we found in the previous section.

In general, the partition function of QCD is not known in a closed analytic form and consequently the zeros are less well understood. If the partition function depends only on $M e^{i \theta / N_{f}}$, then the zeros in the complex $\mu$-plane and the complex $\theta$-plane are related, e.g. the zeros in the complex $\mu$-plane are simply rotated around the origin when varying $\theta$. However, from this relation alone the accumulation of zeros in the complex $\theta$-plane in the vicinity of $\theta=\pi$ and thus Dashen's phenomenon do not follow.

\section{SUMMARY}

We have shown that Dashen's phenomenon is generic for all three $\mathrm{S} \chi \mathrm{SB}$ patterns, $\beta=1,2$ and 4 . By summing the finite volume gauge theory partition functions over all topological charges in the Leutwyler-Smilga regime, we have been able to directly calculate the $\theta$-dependence of the corresponding chiral condensate and topological density. For two flavors, these quantities were shown to be proportional thus establishing a link between chiral and topological properties of the underlying theory. At $\theta=\pi$ the chiral condensate vanishes for degenerate and non-degenerate quark masses and for all three $\beta$. The topological density has a discontinuity for all three values of $\beta$ signaling the first-order phase transition predicted by Dashen. We have illuminated the mechanism by which the phase transition occurs through an inspection of the zeros of the different partition functions.

\section{ACKNOWLEDGMENTS}

We thank P.H. Damgaard and Michel Tytgat for useful conversations and correspondence. The work of G.A. was supported in part by EU TMR grant ERBFMRXCT970122 and by the European network EUROGRID.

\section{APPENDIX: GENERAL $N_{F}$}

We will outline here how to derive the full partition function with $N_{f}>2$ flavors for $\beta=1$. The case $\beta=4$ works out along the same lines and expressions for $\Sigma$ and $\sigma$ follow subsequently. For fixed topological charge, we have 114

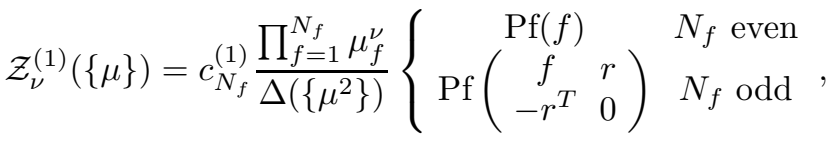

$$
\begin{aligned}
& f^{i j}=\int_{0}^{1} d t t^{2} \frac{I_{\nu-1}\left(t \mu_{i}\right)}{\mu_{i}^{\nu-1}} \frac{I_{\nu}\left(t \mu_{j}\right)}{\mu_{j}^{\nu}}-(i \leftrightarrow j), \\
& r^{i}=\frac{I_{\nu}\left(\mu_{i}\right)}{\mu_{i}^{\nu}} .
\end{aligned}
$$

$c_{N_{f}}^{(1)}$ is a $\nu$-independent constant and $\Delta$ is the Vandermonde determinant. In Ref. [12] sums over topological charge of the following type have been evaluated explicitly:

$$
\mathcal{B}=\sum_{\nu=-\infty}^{\infty} e^{i \nu \theta} I_{\nu+l_{1}}\left(\mu_{1}\right) \cdots I_{\nu+l_{N_{f}}}\left(\mu_{N_{f}}\right) .
$$

We start with even $N_{f}$. When rewriting $\mathrm{Pf}=\sqrt{\operatorname{det}}$ above we can pull the prefactor $\prod_{f=1}^{N_{f}} \mu_{f}^{\nu}$ inside the square root and multiply each $i$ th row of $\operatorname{det} f^{i j}$ with $\mu_{i}^{\nu}$ and each $j$ th column with $\mu_{j}^{\nu}$. We obtain that the factors of $\mu^{\nu}$ in $f^{i j}$ disappear. We can thus apply eqn. (A2) after writing out the Pfaffian since the only $\nu$-dependence left is in the Bessel functions. The results is a Pfaffian over different integrals of a single Bessel function. For $N_{f}$ odd, we only multiply the first $N_{f}$ rows and columns of the matrix of size $N_{f}+1$ inside the Pfaffian. In this way, the matrix elements $r^{i}$ in the last column and row get multiplied only once with $\mu_{i}^{\nu}$, which is precisely what we need to cancel $\mu_{i}^{-\nu}$. We then proceed as for even $N_{f}$.

For $\beta=4$ and $\bar{N}_{f}=2$ we give the full partition function following from eqn. (2.8) for the sake of completeness: 


$$
\begin{gathered}
\mathcal{Z}^{(4)}\left(\theta, \mu_{1}, \mu_{2}\right)=\int_{0}^{2 \pi} \frac{d b}{4 \pi} \int_{0}^{\pi} \frac{d a}{2} \sin (a) \\
\times\left\{\cosh \left[\mu_{\mu \eta}(\theta)\left(e^{i a} T+e^{-i a} T^{-1}\right) / 2\right]+(\mu(b) \leftrightarrow \eta(b))\right\} \\
T=\left[\frac{e^{i \theta} \mu(b)+\eta(b)}{\mu(b)+e^{i \theta} \eta(b)}\right]^{\frac{1}{2}}
\end{gathered}
$$

where $\mu_{\mu \eta}(\theta)$ is the reduced mass of $\mu(b)$ and $\eta(b)$ as in the definition (2.5) and we have first performed the sum over topological charge.

At $\theta=\pi$ the partition function (A3) simplifies drastically and we obtain

$$
\begin{aligned}
\mathcal{Z}^{(4)}\left(\pi, \mu_{1}, \mu_{2}\right) & =\int_{0}^{2 \pi} \frac{d b}{4 \pi} \int_{0}^{\pi} d a \\
& \times \sin (a) \cosh \left[\sin (a)\left(\mu_{1}-\mu_{2}\right) \cos (2 b)\right] \\
& =\frac{\sinh \left(\mu_{1}-\mu_{2}\right)}{\mu_{1}-\mu_{2}} .
\end{aligned}
$$

We can now easily see that the full chiral condensate $\Sigma^{(4)}(\theta)=\frac{1}{2}\left(\Sigma_{1}^{(4)}(\theta)+\Sigma_{2}^{(4)}(\theta)\right)$ vanishes as it is obtained from $\left(\partial_{\mu_{1}}+\partial_{\mu_{2}}\right) \log \mathcal{Z}^{(4)}\left(\pi, \mu_{1}, \mu_{2}\right)$. This is obviously zero for $\mathcal{Z}^{(4)}\left(\pi, \mu_{1}, \mu_{2}\right)$ being a function of $\left(\mu_{1}-\mu_{2}\right)$ only at $\theta=\pi$. The vanishing of $\sigma^{(4)}(\theta)$ can be seen from a similar calculation leading to

$$
\begin{aligned}
0 & =\left.\frac{\partial}{\partial \theta} \mathcal{Z}^{(4)}\left(\theta, \mu_{1}, \mu_{2}\right)\right|_{\theta=\pi}=\int_{0}^{2 \pi} \frac{d b}{4 \pi} \int_{0}^{\pi} d a \quad \text { (A5) } \\
& \times \sin (a) \cos (a)\left(\mu_{1}+\mu_{2}\right) \sinh \left[\sin (a)\left(\mu_{1}-\mu_{2}\right) \cos (2 b)\right],
\end{aligned}
$$

which vanishes because of the first integral. For $\theta=0$ we get from (A3)

$$
\mathcal{Z}^{(4)}\left(0, \mu_{1}, \mu_{2}\right)=\frac{\sinh \left(\mu_{1}+\mu_{2}\right)}{\mu_{1}+\mu_{2}} .
$$

Along with (A4) and by analogy with $\beta=1$ and $\beta=2$ this suggests that $\mathcal{Z}^{(4)}\left(\theta, \mu_{1}, \mu_{2}\right)=\sinh \left(\mu_{12}(\theta)\right) / \mu_{12}(\theta)$ although we cannot prove this. If this is so then the analysis of section III and IV for $\beta=1$ and $\beta=2$ with non-degenerate masses applies equally well to $\beta=4$.

Finally, we display the $\beta=4$ degenerate four-flavor case as an explicit example for $\bar{N}_{f}>2$ :

$$
\begin{aligned}
& \mathcal{Z}^{(4)}(\theta,\{\mu\})=\frac{3}{\mu^{2}}\left(-\frac{I_{1}\left(4 \mu \cos \frac{\theta}{4}\right)}{4 \mu \cos \frac{\theta}{4}}-\frac{I_{1}\left(4 \mu \sin \frac{\theta}{4}\right)}{4 \mu \sin \frac{\theta}{4}}\right. \\
& \left.+\int_{0}^{1} \frac{d t}{2}\left[I_{0}\left(2 \mu \sqrt{1+t^{2}+2 t \cos \frac{\theta}{2}}\right)+\left(\frac{\theta}{2} \rightarrow \frac{\theta}{2}+\pi\right)\right]\right) .
\end{aligned}
$$

It can be most easily obtained using the $\chi \mathrm{RMT}$ correspondence [20].
[1] K.F. Smith et al., Phys. Lett. B 234, 191 (1990); I.S. Altarev et al., Phys. Lett. B 276, 242 (1992).

[2] H. Leutwyler and A. Smilga, Phys. Rev. D 46, 5607 (1992).

[3] J. Gasser and H. Leutwyler, Phys. Lett. B 188, 477 (1987).

[4] J. Verbaarschot, Phys. Rev. Lett. 72, 2531 (1994) [hep-th/9401059].

[5] P. Di Vecchia and G. Veneziano, Nucl. Phys. B 171, 253 (1980).

[6] E. Witten, Ann. Phys. 128, 363 (1980).

[7] A. Smilga and J. Verbaarschot, Phys. Rev. D 51, 829 (1995) [hep-th/9404031.

[8] R. Dashen, Phys. Rev. D 3, 1879 (1971).

[9] M. Creutz, Phys. Rev. D 52, 2951 (1995) [hep-th/9505112].

[10] A.V. Smilga, Phys. Rev. D 59114021 (1999) [hep-ph/9805214].

[11] M.H. Tytgat, Phys. Rev. D 61, 114009 (2000) [hep-ph/9909532].

[12] J. Lenaghan and T. Wilke, Nucl. Phys. B 624, 253 (2002) [hep-th/0108166].

[13] R. Brower, P. Rossi and C. Tan, Nucl. Phys. B 190, 699 (1981); A.D. Jackson, M.K. Sener and J.J.M. Verbaarschot, Phys. Lett. B 387, 355 (1996) [hep-th/9605183]; A.B. Balantekin, Phys. Rev. D 62, 085017 (2000) [hep-th/0007161].

[14] T. Nagao and S.M. Nishigaki, Phys. Rev. D 62, 065006 (2000) [hep-th/0001137.

[15] G. Akemann and E. Kanzieper, Phys. Rev. Lett. 851174 (2000) [hep-th/0001188].

[16] J.J.M. Verbaarschot and T. Wettig, Ann. Rev. Nucl. Part. Sci. 50, 343 (2000) hep-ph/0003017.

[17] P.H. Damgaard, Nucl. Phys. B 556, 327 (1999) [hep-th/9903096].

[18] A.D. Jackson, C.B. Lang, M. Oswald and K. Splittorff, Nucl. Phys. B 616, 233 (2001) hep-th/0108141.

[19] C.N. Yang and T.D. Lee, Phys. Rev. 87404 (1952); Phys. Rev. 87410 (1952).

[20] G. Akemann and P.H. Damgaard, Nucl. Phys. B 576, 597 (2000) [hep-th/9910190]. 